\title{
Mitigation of cross-eye jamming using a dual-polarization array
}

\author{
MA Jiazhi ${ }^{1}$, SHI Longfei ${ }^{1, *}$, XIAO Shunping ${ }^{1}$, and WANG Xuesong ${ }^{1,2}$ \\ 1. State Key Laboratory of Complex Electromagnetic Environmental Effects on Electronics and Information System, \\ National University of Defense Technology, Changsha 410073, China; \\ 2. School of Science, National University of Defense Technology, Changsha 410073, China
}

\begin{abstract}
This paper presents an approach for mitigating the cross-eye jamming using a dual-polarization array. By transmitting a sum beam and a difference beam in two orthogonal polarimetric channels, a synthesized transmitted beam with spatially varying polarization is produced, such that the polarization of the transmitted radar wave varies in azimuth or elevation. Thus, the phases of the signals received on the two antennas of a cross-eye jammer become unequal, and an additional phase difference is introduced to disrupt the $180^{\circ}$ phase shifting in the retrodirective loop of the jammer. By means of beam scanning in a small angular range, the optimal beam steering configuration can be found to maximize the phase error for the mitigation of cross-eye jamming. As a result, the jamming performance of the cross-eye jammer degrades largely. Theoretical analysis and simulation results indicate that the proposed method is valid and feasible.
\end{abstract}

Keywords: cross-eye jamming, monopulse radar, spatially varying polarization, insertion phase, beam scanning.

DOI: $10.21629 / J S E E .2018 .03 .06$

\section{Introduction}

Retrodirective cross-eye jamming (RCJ) is an angular deception technique that deceives monopulse radars into tracking in the wrong direction [1,2]. A retrodirective loop $(\mathrm{RL})$ is the basic structure used to implement a cross-eye jammer, as shown in Fig. 1. In an RL, the signal transmitted by a radar system is received by the two antennas and processed to produce a jamming signal comprising a pair of inverted-amplitude signals, which are ideally $180^{\circ}$ out of phase.

In recent years, the research about cross-eye jamming has been a much concerned research area $[1-11]$. An extended analysis of RCJ based on a single RL was firstly

\footnotetext{
Manuscript received July 31, 2017.

*Corresponding author.

This work was supported by the National Natural Science Foundation of China (61490692; 61401488).
}

presented in [3], and the effect of the platform skin return in RCJ was investigated in [4]. To further improve the jamming effectiveness of RCJ, multiple-element retrodirective cross-eye jamming (MRCJ) was proposed in [511]. In [5], an analysis of MRCJ based on one- and twodimensional arrays was presented. Liu et al. proposed a new rotating implementation structure of MRCJ [6]. Liu et al. presented a comprehensive analysis of MRCJ when the effects of platform exists [7-9]. Du Plessis further investigated the path-length effect between multiple RLs and the jamming gain in MRCJ [10,11].

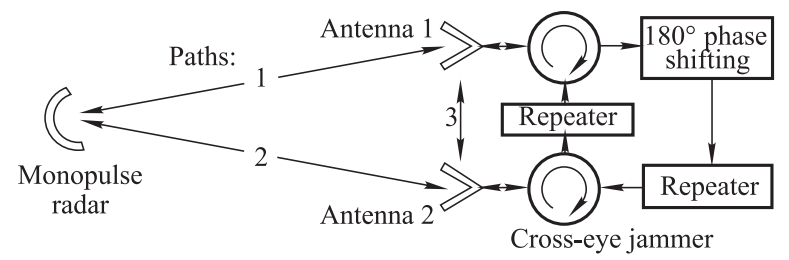

Fig. 1 RL of a cross-eye jammer

However, there are few studies in the literature that have focused on how to detect and mitigate RCJ, and only one patent [12] can be found on this topic. In [12], angle estimates were obtained by finding the maximum response during conical scanning (in an approach called sequential lobing [1]). If these estimates significantly diverge from the monopulse measurements, it is concluded that crosseye jamming is occurring, since RCJ has no influence on the sum beam [4]. Sequential lobing is then performed in place of monopulse processing to counteract the RCJ. However, because of the poor tracking performance of sequential lobing for a fluctuating target [1], the antijamming performance of the method proposed in [12] is limited, although it allows cross-eye jamming to be detected.

In previous analyses of RCJ and MRCJ, antenna polarization was not considered [1-11]; therefore, the antenna insertion phase in the RL, which is strongly related to the 
antenna polarization $[13,14]$, was also neglected. If the polarization of the transmitted radar wave varies in the $3 \mathrm{~dB}$ main-lobe, different insertion phases will arise on the two jammer antennas, thereby introducing an additional phase error into the $180^{\circ}$ phase shifting in the RL. It is known that a cross-eye jammer functions best when there is a $180^{\circ}$ phase difference between the signals radiated from the two antennas; consequently, the introduction of such a phase error would shift the relative phase and thus reduce the effectiveness of the jammer. Because RCJ and MRCJ are always used in jamming missiles, the proposed approach is very suitable for the antijamming of the monopulse radar seekers.

In what follows, Section 2 discusses how to produce a transmitted beam with a spatially varying polarization. Section 3 demonstrates the antijamming principle. Section 4 gives some discussions about the proposed method. Section 5 presents the simulation results. Finally, Section 6 concludes the work.

\section{Producing the transmitted beam}

The monopulse technique is an established method of radar tracking for estimating the angle of a target [1]. By comparing the difference and sum of the signals returned from two sub-beams in each angular dimension, the monopulse ratio (MR) is obtained, which varies linearly in the $3 \mathrm{~dB}$ main-lobe. If we apply the monopulse concept to the transmission from a dual-polarization array with vertical (V) and horizontal $(\mathrm{H})$ polarizations, a transmitted beam with a polarization that is spatially varying in azimuth or elevation can be produced. Fig. 2 illustrates the implementation of such an array that can transmit with a spatially varying polarization.

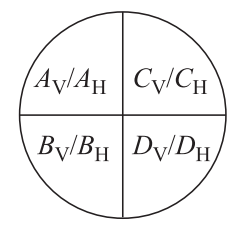

(a) Dual-polarization monopulse radar array partitioning

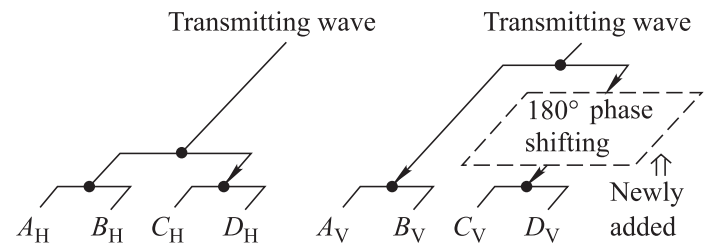

(b) Implementation of the generation of a transmitted beam with a spatially varying polarization

Fig. 2 Implementation of an array that can transmit with a spatially varying polarization
Fig. 2(a) shows that the elements of the array are equally arranged and are divided into four subarrays, which consist typical monopulse arrays for each polarization. In Fig. 2(b), using azimuthal variation as an example, a $180^{\circ}$ phase-shifting block is added on the transmission path for $\mathrm{V}$ polarization, which is similar to the method used to generate the difference channel in the receiving portion of a monopulse system. For simplicity in the following analysis, we assume that the patterns of the array elements for the two orthogonal polarizations are the same; thus, the patterns of the subarrays are also the same (aside from the polarization).

For the system depicted in Fig. 2, the transmission patterns of the two orthogonal polarimetric channels in the azimuth can be modeled as

$$
\begin{aligned}
& P_{\mathrm{tV}}(\theta)=P_{A}(\theta)+P_{B}(\theta)-P_{C}(\theta)-P_{D}(\theta) \\
& P_{\mathrm{tH}}(\theta)=P_{A}(\theta)+P_{B}(\theta)+P_{C}(\theta)+P_{D}(\theta)
\end{aligned}
$$

where $\theta$ is the azimuth angle and $P_{A}(\theta), P_{B}(\theta), P_{C}(\theta)$, and $P_{D}(\theta)$ are the patterns of the subarrays $A, B, C$, and $D$, respectively. Using the polarization angles $(\gamma, \delta)$ [14], the polarization of the transmitted radar wave in the $3 \mathrm{~dB}$ main-lobe of $P_{\mathrm{tH}}(\theta)$ is written as

$$
\tan \gamma \mathrm{e}^{\mathrm{j} \delta}=P_{\mathrm{tV}}(\theta) / P_{\mathrm{tH}}(\theta)=M_{\mathrm{p}}(\theta)
$$

where $M_{\mathrm{p}}(\theta)$ is the MR of the array in the azimuth, which is an approximately linear function of the angle $\theta$ with a monopulse slope of $k_{\mathrm{m}}$. From (3), we can see that the polarization of the radar wave changes linearly, as does the $\mathrm{MR}$, in the $3 \mathrm{~dB}$ main-lobe of the transmitted beam. In other words, a transmitted beam with a spatially varying polarization is formed.

As shown in Fig. 3, to enhance the antijamming performance, an attenuator and a controllable phase shifter are also needed to increase the rate of spatial change of $\gamma$ and to control $\delta$ in (3). Thus, the polarization of the transmitted radar wave in the $3 \mathrm{~dB}$ main-lobe in (3) is modified as follows:

$$
\tan \gamma \mathrm{e}^{\mathrm{j} \delta}=M_{\mathrm{p}}(\theta) \cdot K \mathrm{e}^{\mathrm{j} \delta_{\mathrm{c}}}
$$

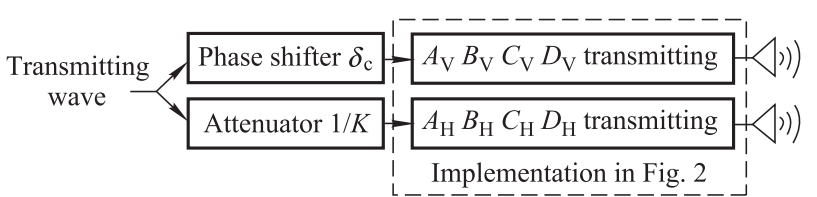

Fig. 3 Flow chart of transmission with a spatially varying polarization

Moreover, by exchanging the sum and difference beams in the two orthogonal polarimetric channels for transmission, the full ranges of $\gamma$ and $\delta$ can be achieved. In Fig. 4, an example is shown of the polarization parameters of an array with $k_{\mathrm{m}}=1.6$ and $K=2$. 


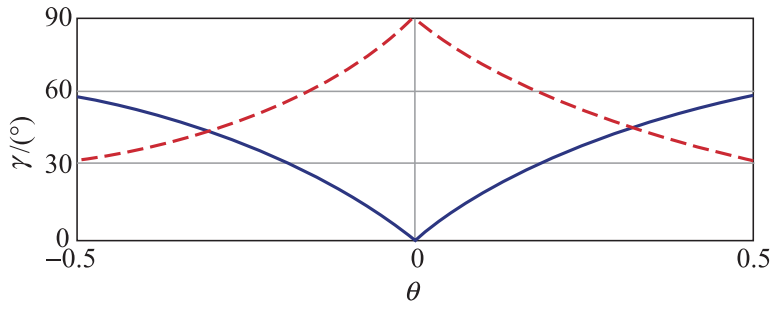

- $: \mathrm{V}$ difference and $\mathrm{H}$ sum; $\quad--: \mathrm{H}$ difference and $\mathrm{V}$ sum.

(a) Variations in $\gamma$

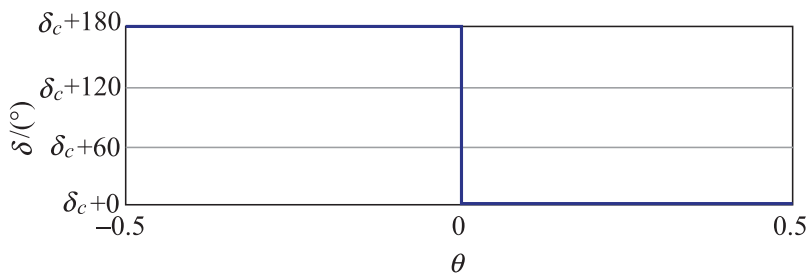

(b) Variations in $\delta$

Fig. 4 Polarization with varying $\theta$ in $3 \mathrm{~dB}$ beamwidth

Unlike traditional dual-polarization radars, such as weather radars, whose transmitted and received polarizations always remain stable in the $3 \mathrm{~dB}$ main-lobe [15], the system proposed in this section is the first to be capable of transmission with a spatially varying polarization. Standard monopulse processing is assumed for radar reception in this paper.

\section{Antijamming principle}

\subsection{Residual phase error}

Consider the case of RCJ with a single RL, as depicted in Fig. 1, the cross-eye jammer operates in two stages. In the receiving stage, the two antennas receive the incident wave from the target radar and generate two corresponding complex voltages. In the transmitting stage, the signal received on one antenna is sent to the other antenna for retransmission, and a $180^{\circ}$ phase shift is introduced on one of the signal paths between the antennas. We let the polarization of the antennas in the RL be denoted by $\boldsymbol{e}_{a}$ (in the previous literature, the polarizations of the antennas in an RL have been assumed to be the same $[1-11])$. When a beam with a spatially varying polarization is used, the polarizations of the incident radar wave are different at the two antennas. We denote them by $\boldsymbol{e}_{1}$ and $\boldsymbol{e}_{2}$. Then, we have

$$
\begin{gathered}
\boldsymbol{e}_{\mathrm{a}}=\left[\begin{array}{ll}
\cos \gamma_{\mathrm{a}} & \sin \gamma_{\mathrm{a}} \mathrm{e}^{\mathrm{j} \delta_{\mathrm{a}}}
\end{array}\right]^{\mathrm{T}} \\
\boldsymbol{e}_{1,2}=\left[\begin{array}{ll}
\cos \gamma_{1,2} & \sin \gamma_{1,2} \mathrm{e}^{\mathrm{j} \delta_{1,2}}
\end{array}\right]^{\mathrm{T}} .
\end{gathered}
$$

Therefore, the normalized complex voltages generated on the jammer antennas in the receiving stage of the jammer are

$$
v\left(\boldsymbol{e}_{1,2}, \boldsymbol{e}_{\mathrm{a}}\right)=\boldsymbol{e}_{1,2}^{\mathrm{T}} \cdot \boldsymbol{e}_{\mathrm{a}}^{*}=\cos \gamma_{1,2} \cos \gamma_{\mathrm{a}}+
$$

$$
\sin \gamma_{1,2} \sin \gamma_{\mathrm{a}} \mathrm{e}^{\mathrm{j}\left(\delta_{1,2}-\delta_{\mathrm{a}}\right)} .
$$

The insertion phases, namely, the phases of the two complex voltages, are defined by the following function [14]:

$$
\begin{gathered}
\phi_{p_{1}, p_{2}}=f\left(\gamma_{1,2}, \delta_{1,2}, \gamma_{\mathrm{a}}, \delta_{\mathrm{a}}\right)=\arg \left[v\left(\boldsymbol{e}_{1,2}, \boldsymbol{e}_{\mathrm{a}}\right)\right]= \\
\tan ^{-1} \frac{\sin \gamma_{1,2} \sin \gamma_{\mathrm{a}} \sin \left(\delta_{1,2}-\delta_{\mathrm{a}}\right)}{\cos \gamma_{1,2} \cos \gamma_{\mathrm{a}}+\sin \gamma_{1,2} \sin \gamma_{\mathrm{a}} \cos \left(\delta_{1,2}-\delta_{\mathrm{a}}\right)} .
\end{gathered}
$$

Thus, the phase difference between the two insertion phases, called the residual phase error (RPE) [14], can be expressed as

$$
\phi_{r}=\phi_{p_{2}}-\phi_{p_{1}}
$$

In addition, the polarization efficiencies $p$ of the two antennas, sometimes referred to as the polarization mismatch losses, are

$$
p_{1,2}=\left|v\left(\boldsymbol{e}_{1,2}, \boldsymbol{e}_{\mathrm{a}}\right)\right|^{2} .
$$

In the transmitting stage of the cross-eye jammer, to ensure a suitable jamming-to-signal ratio (JSR), the transmitting amplifiers always operate in the saturated state. Therefore, as long as the signal strength of the incident radar wave received by the two jammer antennas is higher than the receiver sensitivity of the jammer, the polarization mismatch losses in the receiving stage have no influence on the RCJ performance, but the RPE still exists. Because the polarization in the $3 \mathrm{~dB}$ main-lobe in radar receiving is spatially invariant, the insertion phases of the signals that are transmitted by the two jammer antennas (which have the same polarization) and received by the radar are equal; thus, an RPE is introduced only in the $180^{\circ}$ phase shift in the receiving stage of the jammer.

In the previous literature, the RPE was often studied in the analysis of interferometers composed of multiple receiving antennas [13]. For interferometers, antennas with different polarization properties have been shown to exhibit different phase responses to the polarization of incident waves, which may result in significant measuring error. Unlike in previous RPE research, in this paper, the polarizations of the antennas are the same, whereas that of the incident wave is spatially varying. We assume that the polarization of the wave are

$$
\begin{gathered}
\gamma_{1,2}=\gamma \mp \Delta \gamma \\
\delta_{1,2}=\delta=\delta_{\mathrm{c}} \text { or } \delta_{\mathrm{c}}+\pi .
\end{gathered}
$$

Then, the first-order approximation of the RPE is

$\phi_{\mathrm{r}}=f\left(\gamma+\Delta \gamma, \delta_{2}, \gamma_{\mathrm{a}}, \delta_{\mathrm{a}}\right)-f\left(\gamma-\Delta \gamma, \delta_{1}, \gamma_{\mathrm{a}}, \delta_{\mathrm{a}}\right) \approx$

$$
\frac{\partial f\left(\gamma, \delta, \gamma_{\mathrm{a}}, \delta_{\mathrm{a}}\right)}{\partial \gamma} \cdot 2 \Delta \gamma=
$$




$$
\frac{-\sin \left(\delta-\delta_{\mathrm{a}}\right) \cdot 2 \Delta \gamma}{\cos ^{2} \gamma \cot \gamma_{\mathrm{a}}+\sin 2 \gamma \cos \left(\delta-\delta_{\mathrm{a}}\right)+\sin ^{2} \gamma \tan \gamma_{\mathrm{a}}}
$$

If RCJ is detected using the method proposed in [12], then the polarization of the jammer antennas $\left(\gamma_{\mathrm{a}}, \delta_{\mathrm{a}}\right)$ can be estimated from the received jamming signals. Therefore, the absolute value of the RPE can be maximized by tuning $\gamma$ and $\delta$. To find the maximum $\left|\phi_{\mathrm{r}}\right|$, we consider

$$
\frac{\partial^{2} f\left(\gamma, \delta, \gamma_{\mathrm{a}}, \delta_{\mathrm{a}}\right)}{\partial \gamma^{2}}=0
$$

and get

$$
\tan 2 \gamma \cdot \cot 2 \gamma_{\mathrm{a}}=\cos \left(\delta-\delta_{\mathrm{a}}\right) .
$$

Because $\left(\delta-\delta_{\mathrm{a}}\right)$ should not be 0 (otherwise, $\phi_{\mathrm{r}}$ is equal to 0 , as seen from (8)), we can see that when $\gamma=\pi / 2-\gamma_{\mathrm{a}}$ and $\left(\delta-\delta_{\mathrm{a}}\right) \rightarrow \pi$, (14) is approximately satisfied. Then, according to (4), by adjusting the antenna orientation and controlling the phase $\delta_{\mathrm{c}}$ in Fig. 3, a phase error corresponding to the maximum $\left|\phi_{\mathrm{r}}\right|$ can be introduced into the $180^{\circ}$ phase shift in the RL to mitigate the RCJ.

For typical RCJ scenarios, the included angles between the two jammer antennas versus the distance between the radar and the cross-eye jammer are shown in Fig. 5 for different antenna separation distances. And in Fig. 5, the $3 \mathrm{~dB}$ beamwidth of the radar is $5.8^{\circ}$ in simulation. Taking the array considered in Fig. 4 as an example, by comparing Fig. 4(a) and Fig. 5, we can see that when the distance is $3 \mathrm{~km}$, the included angle between the two jammer antennas is approximately 0.05 times the $3 \mathrm{~dB}$ beamwidth and $2 \Delta \gamma$ is approximately $7.25^{\circ}$. If the distance between the jammer and the target becomes closer, the polarization difference $2 \Delta \gamma$ will be larger as well.

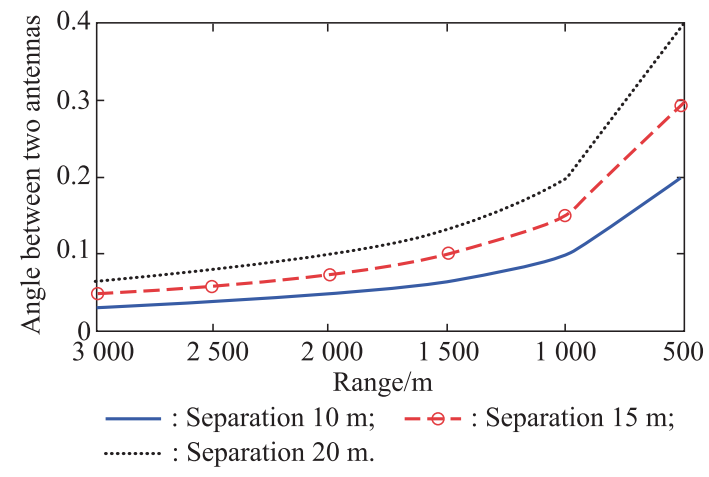

Fig. 5 Included angles of the two jammer antennas during tracking versus the distance between the radar and the jammer

Based on (8), (9) and (13), we can compute $\left|\phi_{\mathrm{r}}\right|$ for the cases in which the jammer antennas have linear and circular polarizations. The simulated results are shown in Fig. 6 and Fig. 7, respectively. We can see that the first-order approximation of $\left|\phi_{\mathrm{r}}\right|$ in (13) is very close to the actual value of $\left|\phi_{\mathrm{r}}\right|$ in (9) and that the region in which $\left|\phi_{\mathrm{r}}\right|$ is maximized (the region of $\gamma$ and $\delta$ for which $\left|\phi_{\mathrm{r}}\right| \geqslant 15^{\circ}$ ) conforms to the principle based on which we find the maximum $\left|\phi_{\mathrm{r}}\right|$, as in (14).

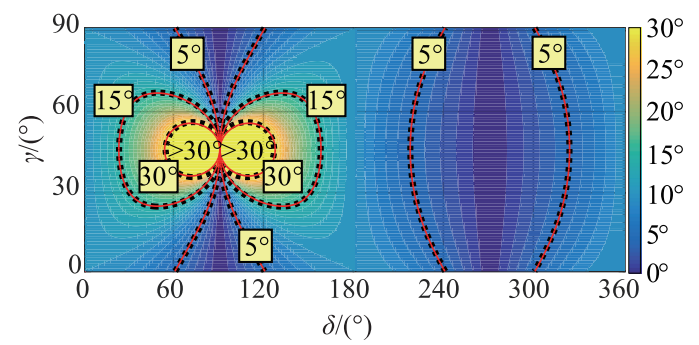

(a) $\gamma_{\mathrm{a}}=45^{\circ}$ and $\delta_{\mathrm{a}}=270^{\circ}$

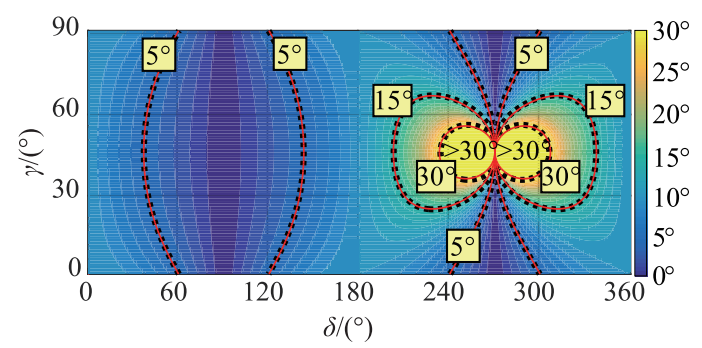

(b) $\gamma_{\mathrm{a}}=45^{\circ}$ and $\delta_{\mathrm{a}}=90^{\circ}$

---- : Numerical; _ : First order.

Fig. 6 Contours of $\left|\phi_{\mathrm{r}}\right|$ when the jammer antennas are circularly polarized $\left(2 \Delta \gamma=7.25^{\circ}\right)$

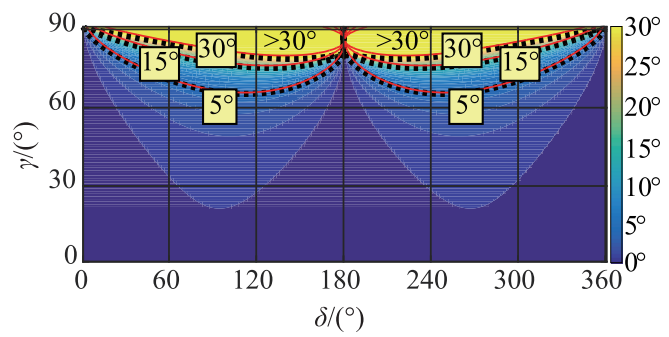

(a) $\gamma_{\mathrm{a}}=5^{\circ}$ and $\delta_{\mathrm{a}}=0^{\circ}$

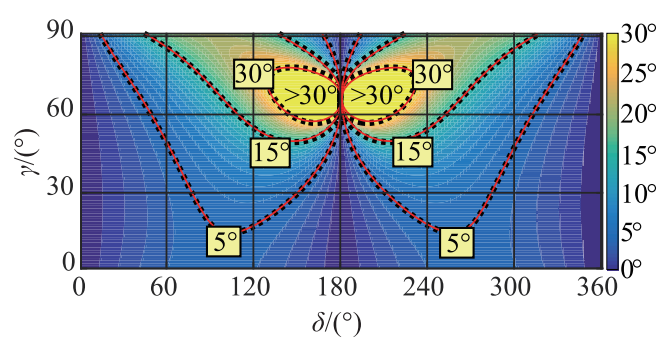

(b) $\gamma_{\mathrm{a}}=25^{\circ}$ and $\delta_{\mathrm{a}}=0^{\circ}$

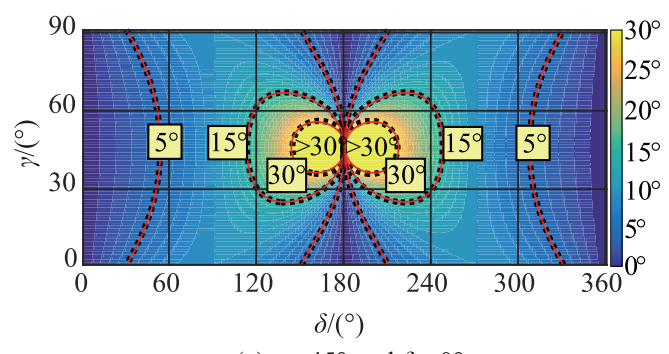

(c) $\gamma_{\mathrm{a}}=45^{\circ}$ and $\delta_{\mathrm{a}}=0^{\circ}$ 


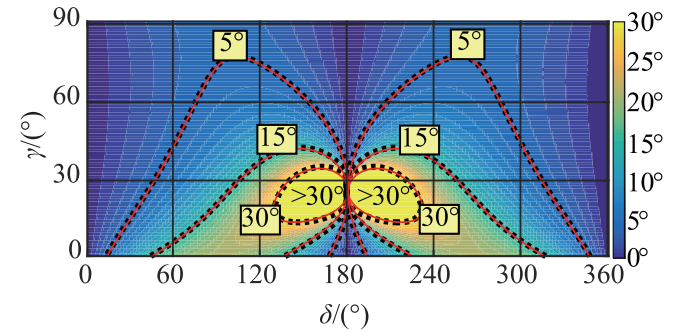

(d) $\gamma_{\mathrm{a}}=65^{\circ}$ and $\delta_{\mathrm{a}}=0^{\circ}$

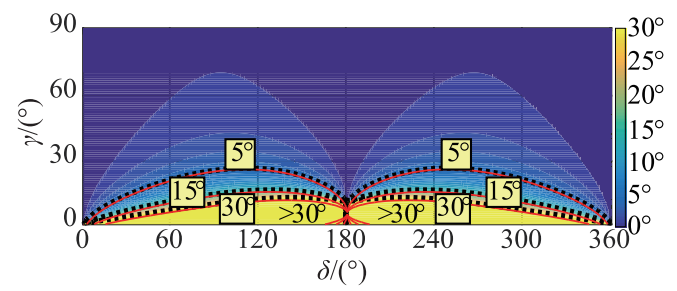

(e) $\gamma_{\mathrm{a}}=85^{\circ}$ and $\delta_{\mathrm{a}}=0^{\circ}$

---- : Numerical; — : First order.

Fig. 7 Contours of $\left|\phi_{\mathbf{r}}\right|$ when the jammer antennas are linearly polarized $\left(2 \Delta \gamma=7.25^{\circ}\right)$

\subsection{Beam steering and beam scanning}

To increase the parameter tolerance, $\gamma$ and $\delta$ should be modified to lie near the center of the contours for a specific $\left|\phi_{\mathrm{r}}\right|$ value, as shown in Fig. 6 and Fig. 7. We define two variables $\delta_{\min }$ and $\delta_{\max }$ :

$$
\begin{gathered}
\arg \delta_{\min }=\min _{\delta-\delta_{\mathrm{a}}>\pi} \delta, \\
\text { s.t. }\left|\phi_{\mathrm{r}}\right|=\phi_{\mathrm{s}}, \quad \gamma=\pi / 2-\gamma_{\mathrm{a}} \\
\arg \delta_{\max }=\min _{\delta-\delta_{\mathrm{a}}>\pi} \delta, \\
\text { s.t. }\left|\phi_{\mathrm{r}}\right|=\phi_{\mathrm{s}}, \quad \gamma=\pi / 2-\gamma_{\mathrm{a}}
\end{gathered}
$$

where $\phi_{\mathrm{S}}$ is the RPE set by us to effectively mitigate the distortion of the phase front caused by the RCJ signal. Then, the modified transmitted polarization in the direction of the center of the two jammer antennas $\left(\gamma_{\mathrm{t}}, \delta_{\mathrm{t}}\right)$ should satisfy

$$
\begin{gathered}
\gamma_{\mathrm{t}}=\pi / 2-\gamma_{\mathrm{a}} \\
\delta_{\mathrm{t}}=\delta_{\min }+\left(\delta_{\max }-\delta_{\min }\right) / 2 .
\end{gathered}
$$

Because the actual angle of the target in the $3 \mathrm{~dB}$ mainlobe is unknown when RCJ is present, the transmitted polarization in the direction of the center of the two jammer antennas is also unknown. However, if we perform beam scanning in the angular region of the $3 \mathrm{~dB}$ main-lobe by means of multiple snapshots, one or more snapshots will fall in the polarization region with the maximal $\left|\phi_{\mathrm{r}}\right|$. Therefore, by clustering these monopulse angle measurements and comparing them with the results of sequential lobing, a correct estimate of the target angle can be obtained with a decreased data rate. For practical applications, the minimum beam-scanning step necessary to ensure correct angle estimation should be determined.
As shown in Fig. 6 and Fig. 7, the minimum beamscanning step is actually the length of the projection of the polarization region with a specific $\left|\phi_{\mathrm{r}}\right|$ onto the vertical axis (the $\gamma$ axis). Thus, we define $\gamma_{\min }$ and $\gamma_{\max }$ :

$$
\begin{gathered}
\arg \gamma_{\min }=\min _{\gamma}, \quad \text { s.t. }\left|\phi_{\mathrm{r}}\right|=\phi_{\mathrm{s}}, \quad \delta=\delta_{\mathrm{t}} \\
\arg \gamma_{\max }=\max _{\gamma}, \quad \text { s.t. }\left|\phi_{\mathrm{r}}\right|=\phi_{\mathrm{s}}, \quad \delta=\delta_{\mathrm{t}} .
\end{gathered}
$$

Taking Fig. 6(a) as an example, Fig. 8 shows the meanings of these parameters in (15) $-(20)$.

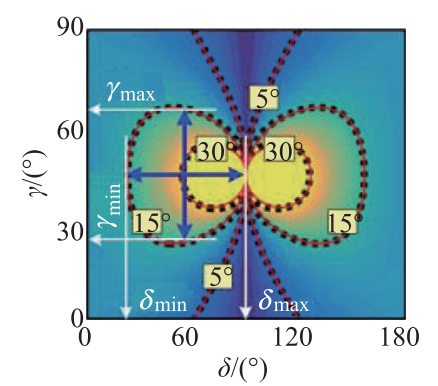

Fig. 8 Explaination of the parameters in deriving the minimum beam-scanning step

Then, the minimum beam-scanning step can be written as

$$
\theta_{\text {step }}=\frac{\theta_{\mathrm{bw}}}{2 k_{\mathrm{m}}}\left|\tan \gamma_{\max }-\tan \gamma_{\text {min }}\right|
$$

where $\theta_{\mathrm{bw}}$ is the $3 \mathrm{~dB}$ beamwidth and $k_{\mathrm{m}}$ is the slope of the monopulse ratio. Taking the array considered in Fig. 4 as an example, the minimum beam-scanning steps for different relative distances between the jammer and the radar are shown in Fig. 9. We can see that the minimum beamscanning step increases as the distance between the radar and the jammer decreases. The minimum beam-scanning steps when $\gamma_{a}>45^{\circ}$ are not plotted because of their similarity.

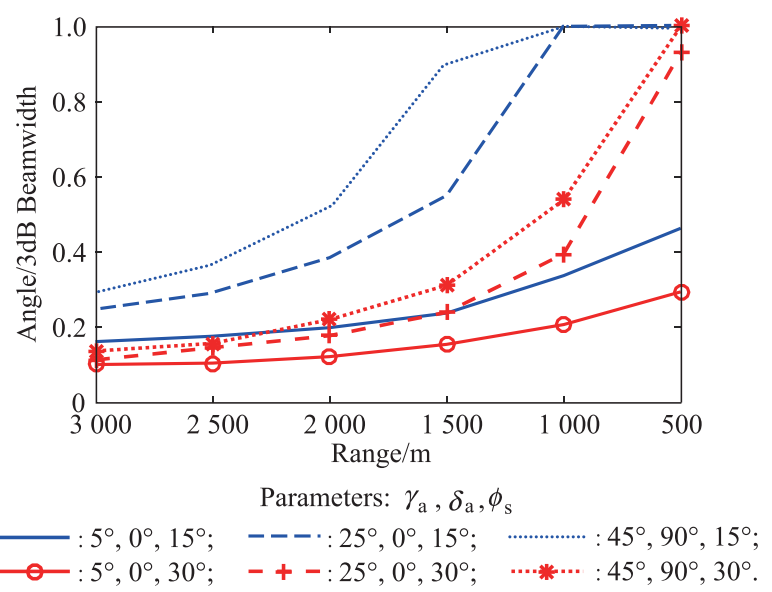

Fig. 9 Minimum beam-scanning steps required under different parameter conditions when the jammer antenna separation distance is $15 \mathbf{m}$ 
Fig. 10 shows the basic procedure of the beam scanning, the beam steering of the radar should be switched periodically between the steering position 1 and the steering position 2. To get more data, the beam scanning speed should be as faster as possible, which will add extra burdens to the beam steering control system in the radar.

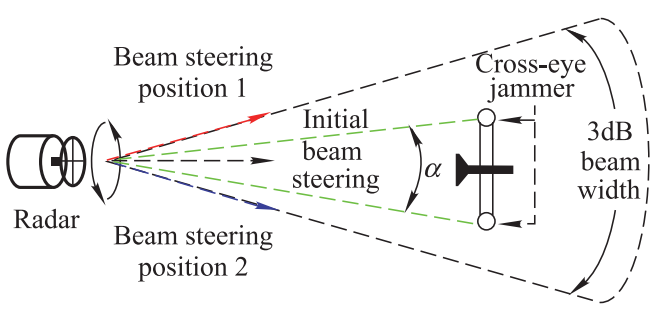

Fig. 10 Basic procedure of the beam scanning

\section{Discussion}

In Fig. 6 and Fig. 7, the peak RPE appears to occur in areas where the incident wave is nearly cross-polarized with respect to the jammer antenna polarization. The following question naturally arises: is inducing an RPE as proposed in this paper more important than simply modifying the radar to be cross-polarized with respect to the jammer to reduce the JSR without the need to transmit with a spatially varying polarization?

In fact, there are two factors limiting the latter approach. First, because the distance between the radar and the jammer is always short, the scenario of RCJ or MRCJ is highly dynamic; the jammer polarization parameter $\gamma_{\mathrm{a}}$ may change rapidly as the relative attitude changes. There are two ways to modify the radar polarization parameter $\gamma$ : the first is to adjust the complex ratio between the two orthogonal polarimetric channels, the second is to use polarization-agile (or polarization reconfigurable) antennas. However, the first method is limited by the rate of change of the magnitude of the complex ratio for transmission (because the transmitting amplifiers of the radar system always operate in the saturated state), and the second method can only provide a few discrete polarization states [15]. Second, because the radar polarization should be as orthogonal as possible to the jammer polarization, the polarization parameter tolerance of the latter approach is small.

In contrast, rapid modification of $\gamma$ can be achieved in the case of transmission with a spatially varying polarization by using a dual-polarization phased array. Moreover, the parameter tolerance of the proposed method is not strict. As shown in Fig. 6, an available range of $\gamma$ is about $40^{\circ}$ when $\gamma_{\mathrm{a}}=45^{\circ}$ and $\delta_{\mathrm{a}}=90^{\circ}\left(\left|\phi_{\mathrm{r}}\right|>15^{\circ}\right)$.

\section{Simulations}

The antijamming performance of the proposed method is evaluated in this section. The scenario parameters in the simulations are typical of a missile engaging an aircraft:

(i) Radar carrier frequency: $15 \mathrm{GHz}$;

(ii) Radar $3 \mathrm{~dB}$ beamwidth: $5.8^{\circ}$;

(iii) The parameters in (4): $K=2$;

(iv) Jammer range: $3 \mathrm{~km}$;

(v) Jammer polarization: circular polarization $\left(\gamma_{\mathrm{a}}=\right.$ $45^{\circ}$ and $\delta_{\mathrm{a}}=270^{\circ}$ );

(vi) Separation distance between the jammer antennas located on the wingtips: $15 \mathrm{~m}$.

The examples of the jammer of RCJ and MRCJ are plotted in Fig. 11.

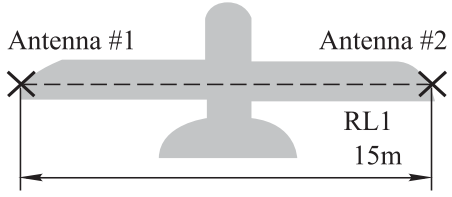

(a) RCJ jammer

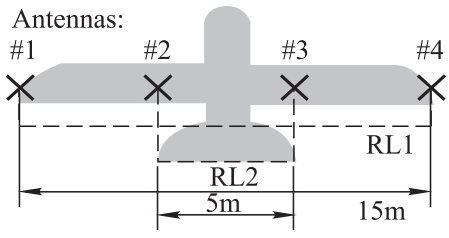

(b) MRCJ jammer

Fig. 11 Antenna separations for cross-eye jammers

According to [4,8], the angle of the signals composed of the jamming signals of RCJ or MRCJ and the target returns can be written as

$$
\theta^{\prime}=G_{\mathrm{Mn}} \cdot \alpha
$$

where $\alpha$ is the included angle between the two jammer antennas located on the wingtips as shown in Fig. 10, and $G_{\mathrm{Mn} n}$ is the median total cross-eye gain (MTCEG).

As in Fig. 10, when the radar scans from the beam steering position 1 to the beam steering position 2, the MTCEGs for the RCJ jammer in Fig. 11(a) under different parameter conditions are plotted in Fig. 12. the parameter JSR $\mathrm{Sini}$ means the initial jamming to signal ratio of RCJ, $\pi+\phi$ means the $180^{\circ}$ phase shifting in the RL when system phase error exists, and $a$ is the transmitting relative amplitude ratio between the two jammer antennas.

Fig. 13 shows the MTCEGs for the MRCJ jammer in a beam scanning cycle under different parameter conditions. The parameters $a_{1}$ and $a_{2}$ are the transmitting relative amplitude ratio between the two jammer antennas in RL 1 and RL 2. In addition, we assume that the total phase difference between RL 1 and RL 2 is $30^{\circ}$.

From Fig. 12 and Fig. 13, it is obvious that when the beam points to the 0.4 times of the $3 \mathrm{~dB}$ beamwidth, the 
MTCEGs of RCJ as well as that of MRCJ decrease largely, which indicates that the proposed approach for mitigating RCJ and MRCJ is valid and feasible.

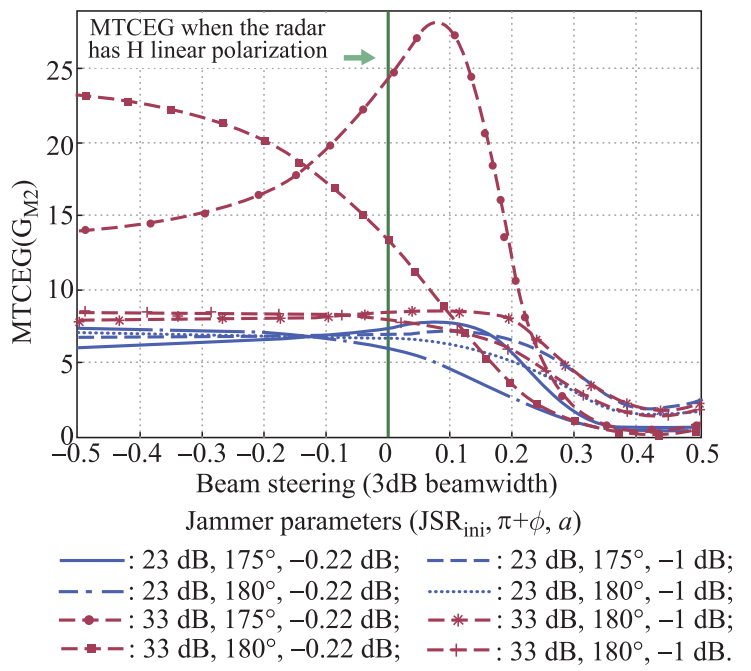

Fig. 12 MTCEG of RCJ in a scanning cycle

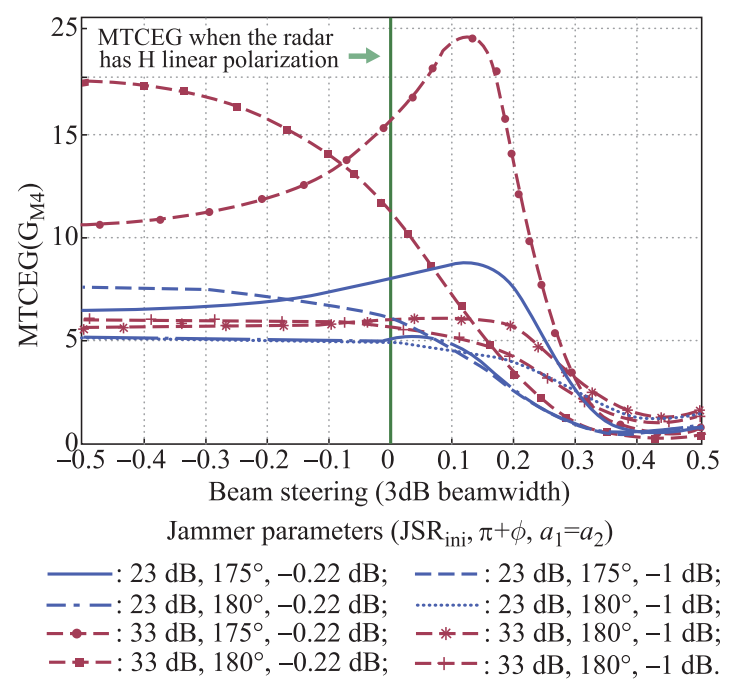

Fig. 13 MTCEG of MRCJ in a scanning cycle

\section{Conclusions}

With a dual-polarization array, an antijamming approach for RCJ and MRCJ is presented in this paper. By transmitting with a spatially varying polarization and beam scanning, a large phase error can be introduced into the $180^{\circ}$ phase shifting performed in the RL, causing the cross-eye jammer to act as a beacon. Thus, the increase in the JSR induced by the attenuator in one of the two polarimetric channels, as shown in Fig. 3, can be ignored. Moreover because the polarization of the transmitted beam is spatially varying, the proposed method can also prevent cross-polarized jamming by disrupting the estimation of the radar polarization for the jammer.

In addition, another merit of the proposed approach is low computation complexity. The main calculation of the proposed approach is estimating the jamming polarization. Because there are many methods that can be used in estimating the wave polarization [16,17], we do not discuss it in this paper.

\section{References}

[1] SHERMAN S M, BARTON D K. Monopulse principles and techniques. 2nd ed. Norwood: Artech House, 2011.

[2] WANG C H, HUANG T M. Cross-eye jamming of monopulse radar. Proc. of the IEEE Waveform Diversity Design, 2007: $209-213$.

[3] DU PLESSIS W P, ODENDAAL J W, JOUBER J. Extended analysis of retrodirective cross-eye jamming. IEEE Trans. on Antennas and Propagation, 2009, 57(9): 2803-2806.

[4] PLESSIS W P D. Platform skin return and retrodirective crosseye jamming. IEEE Trans. on Aerospace and Electronic Systems, 2012, 48(1): 490-501.

[5] HARWOOD N M, DAWBER W N, KLUCKERS V A, et al. Multiple-element cross-eye jamming. IET Radar Sonar \& Navigation, 2007, 1(1): $67-73$.

[6] LIU S Y, DONG C X, XU J, et al. Analysis of rotating crosseye jamming. IEEE Antennas and Wireless Propagation Letters, 2015, 14: 939-942.

[7] LIU T P, LIU Z, LIAO D P, et al. Performance analysis of multiple-element retrodirective cross-eye jamming based on linear array. IEEE Trans. on Aerospace and Electronic Systems, 2015, 51(3): $1867-1876$.

[8] LIU T P, LIU Z, LIAO D P, et al. Platform skin return and multiple-element linear retrodirective cross-eye jamming. IEEE Trans. on Aerospace and Electronic Systems, 2016, 52(2): $1867-1875$.

[9] WANG C Y, HE Z Y, GONG J. Analysis of retro-directive cross-eye jamming for multiple elements. Systems Engineering and Electronics, 2017, 39(7): 1457-1463. (in Chinese)

[10] DU PLESSIS W P. Path-length effects in multi-loop retrodirective cross-eye jamming. IEEE Antennas and Wireless Propagation Letters, 2015, 15: 626-629.

[11] DU PLESSIS W P. Cross-eye gain in multiloop retrodirective cross-eye jamming. IEEE Trans. on Aerospace and Electronic Systems, 2016, 52(2): $875-882$.

[12] CHANDLER C A. Patent: cross-eye jamming detection and mitigation. [2017-07-30]. http://patents.justia.com/patent/ 7843376.

[13] SEIFER A D. Impact of antenna-polarization response on direction of arrival measurement. IEEE Trans. on Antennas and Propagation, 1998, 34(1): 319-329.

[14] LAWRENCE D E. Antenna insertion phase variation with polarization and methods for compensation. IEEE Trans. on Antennas Propagation, 2015, 62(11): 5715-5722.

[15] LEE S W, SUNG Y J. Polarization reconfigurable aperture-fed patch antenna and array. IEEE Access, 2016, 4: 1510-1517.

[16] MA J Z, SHI L F, LI Y Z, et al. Angle estimation of extended targets in main lobe interference with polarization filtering. IEEE Trans. on Aerospace and Electronic Systems, 2017, 53(1): $169-189$.

[17] ZHAO J, TAO H H. Quaternion based joint DOA and polarization parameters estimation with stretched three-component electromagnetic vector sensor array. Journal of Systems Engineering and Electronics, 2017, 28(1): 1-9. 


\section{Biographies}

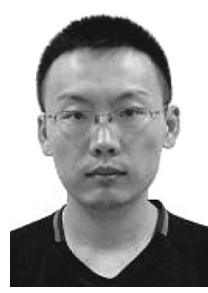

MA Jiazhi received his B.E. and M.E. degrees in control engineering from the Aviation University, Changchun, China, in 2010 and 2013, respectively. $\mathrm{He}$ is currently earning his Ph.D. degree from National University of Defense Technology. His current research interests include radar signal processing and radar polarimetry.

E-mail: jzmanudt@163.com

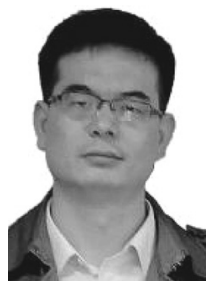

SHI Longfei received his B.E. and Ph.D. degrees in electronic engineering from National University of Defense Technology (NUDT), Changsha, China, in 2002 and 2007, respectively. He is an associate professor at NUDT. His current research interests include digital signal processing and radar polarimetry.

E-mail: longfei_shi@sina.com

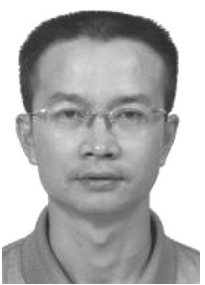

XIAO Shunping received his B.E. and Ph.D. degrees in electronic engineering from National University of Defense Technology (NUDT), Changsha, China, in 1986 and 1995, respectively. He is a professor at NUDT. His current research interests include polarimetric radar information processing.

E-mail: xiaoshunping@nudt.edu.cn

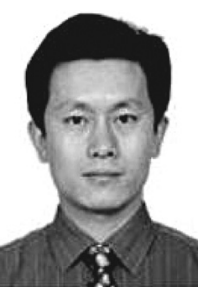

WANG Xuesong received his B.E. and Ph.D. degrees in electronic engineering from National University of Defense Technology (NUDT), Changsha, China, in 1994 and 1999, respectively. He is a professor at NUDT. His current research interests include polarimetric radar information processing and target recognition.

E-mail: wxs1019@vip.sina.com 